\title{
Mining Residue Contacts in Proteins Using Local Structure Predictions
}

\author{
Mohammed J. Zaki ${ }^{\dagger}$, Shan Jin ${ }^{\dagger}$, Chris Bystroff ${ }^{\ddagger}$, \\ ${ }^{\dagger}$ Computer Science Department, ${ }^{\ddagger}$ Biology Department \\ Rensselaer Polytechnic Institute, Troy, NY 12180 \\ zaki@cs.rpi.edu, bystrc@rpi.edu, jins@cs.rpi.edu
}

\begin{abstract}
In this paper we develop data mining techniques to predict $3 D$ contact potentials among protein residues (or amino acids) based on the hierarchical nucleationpropagation model of protein folding. We apply a hybrid approach, using a Hidden Markov Model to extract folding initiation sites, and then apply association mining to discover contact potentials. The new hybrid approach achieves accuracy results better than those reported previously.
\end{abstract}

\section{Introduction}

Today we are witnessing a paradigm shift in predicting protein structure from its known amino acid sequence $\left(a_{1}, a_{2}, \cdots, a_{n}\right)$. The traditional or $\mathrm{Ab}$ initio folding method employed first principles to derive the 3D structure of proteins. However, even though considerable progress has been made in understanding the chemistry and biology of folding, the success of ab initio folding has been quite limited. Instead of simulation studies, an alternative approach is to employ learning from examples using a database of known protein structures. For example, the Protein Data Bank (PDB) records the 3D coordinates of the atoms of thousands of protein structures. Most of these proteins cluster into around 700 fold-families based on their similarity. It is conjectured that there will be on the order of 1000 fold-families for the natural proteins. The PDB thus offers a new paradigm to protein structure prediction by employing data mining methods like clustering, classification, association rules, hidden Markov models, etc.

A fascinating property of protein chains is that they spontaneously and reproducibly fold into complex threedimensional globules when placed in an aqueous solution. The sequence of amino acids making up the polypeptide chain contains, encoded within it, the complete building instructions. This self-organization cannot occur by a random conformational search for the lowest energy state, since such a search would take millions of years, while proteins fold in milliseconds. In recent years, a combination of molecular biological and biophysical techniques have dissected the folding process into fast and slow components which localize to certain parts of the protein sequence.

Some small, fast-folding regions of the molecule may be identified by their sequence alone. A library of short sequence patterns that fold fast has been compiled by cluster analysis of the database of known protein structures (the I-sites Library, [1]). In this work, similar short sequences that mapped to the same local structure in different proteins were deemed to be autonomous folding units, and the short sequences were compiled into patterns or "profiles" which could then be used to predict whether or not a segment of the protein would tend to fold independently of the rest of the molecule. Cross-validation showed a strong statistical significance to the predictions made by the profiles, and later NMR studies showed that some peptides predicted to fold in isolation actually did so [10]. Peptides with a strong tendency to fold independently constitute about $30 \%$ of the amino acid residues in protein sequences. The formation of independent folding units (I-sites motifs) is the first level of self-organization in the folding process: the "initiation."

These short motifs occur in proteins of widely differing topology, and so cannot contain sufficient information to define the overall, global fold of the protein molecule. Moreover, they are too short to be the fast-folding regions found by experimental dissection. There must be a higher level of self-organization which dictates how the short pieces come together to form larger, longer globular domains. The rules defining the propagation of structure along the chain, starting from the sites of initiation, have been extracted from the database of known protein structures and formalized as a hidden Markov model (HMM), called HMMSTR [2] (or "hamster"), discussed further below. HMMSTR models the interactions between adjacent short regions of the sequence, attempting to model the second level of self-organization: "propagation" of structure along the sequence.

The I-sites Library models the initiation sites of folding, and the new HMM models interactions between those sites. But HMMSTR [2] is a network of connections between Isites motifs, and thus simultaneously models both folding initiation and propagation. The two levels of complexity, not discretely defined but smoothly intermingled, are represented in the HMM as variable degrees of branching. Unbranched segments are initiations sites, whose probabilities depend simultaneously on short contiguous segments of the sequence, while branching and cycles represent multiple sequence-dependent ways of extending and linking the initiation sites. Arbitrary levels of complexity may be modeled by including HMMs recursively within overarching HMMs, the latter representing the ways of connecting the output of 
the HMMs it contains. Hidden Markov models are limited to data that can be expressed as one-dimensional sequences of discrete symbols, but there are techniques for overcoming both the discreteness and the one-dimensionality [7].

The next level of complexity in protein folding is called "condensation". In the first few microseconds after introducing the polypeptide chain into an aqueous solution, initiation sites form transient, rapidly-interchanging structures, favoring one or more conformations to varying degrees. These structures propagate along the chain by promoting compatible upstream and downstream conformations, and the resulting transiently-formed substructures encounter each other by through-space diffusion, condensing into larger, ordered globules, as energy dictates. The ordering of these three processes is not discrete but overlapping, and they should therefore be integrated into a single computational model. Modeling of the condensation step given predictions based on the modeling of initiation/propagation is the subject of the present work. A single Markov state prediction implies a local substructure and a single amino acid position within it. Thus, a contact between two Markov states implies a specific mode of condensation between two local substructures to form tertiary structure.

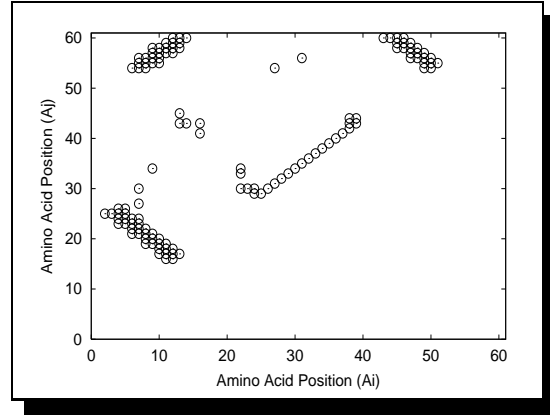

Figure 1. Contact Map (PDB ID 2igd, $N=61$ )

The contact map of a protein (see Figure 1) is a particularly useful representation of protein tertiary structure. Two amino acids in a protein that come into contact with each other form a non-covalent interaction (hydrogen-bonds, hydrophobic effect, etc.). More formally, we say that two residues (or amino acids) $a_{i}$ and $a_{j}$ in a protein are in contact if the 3D distance $\delta\left(a_{i}, a_{j}\right)$ is less than some threshold value $t$ (in this paper we use $t=7 \AA$ as the threshold distance), where $\delta\left(a_{i}, a_{j}\right)=\left|\mathbf{r}_{\mathbf{i}}-\mathbf{r}_{\mathbf{j}}\right|$, and $\mathbf{r}_{\mathbf{i}}$ and $\mathbf{r}_{\mathbf{j}}$ are the coordinates of the $\alpha$-Carbon atoms of amino acids $a_{i}$ and $a_{j}$. We define sequence separation as the distance between two amino acids $a_{i}$ and $a_{j}$ in the amino acid sequence, given as $|i-j|$. A contact map for a protein with $N$ residues is an $N \times N$ binary matrix $C$ whose element $C(i, j)=1$ if residues $i$ and $j$ are in contact, and $C(i, j)=0$ otherwise. The contact map provides a host of useful information. For example, secondary structure can easily be discerned from it. $\alpha$-Helices appear as thick bands along the main diagonal since they involve contacts between one amino acid and its four successors, while $\beta$-Sheets are thin bands parallel or anti-parallel to the main diagonal, etc. However, tertiary structure is not easily found from the contact map. For predicting the elusive global fold of a protein we are usually interested in only those contacts that are far from the main diagonal. In this paper we thus ignore any pair of residues whose sequence separation $|i-j|<4$.

Previous work on contact prediction has employed Neural Networks [3], and statistical techniques based on correlated mutations [6,8]. Recent work by Vendruscolo et al [9] has also shown that it is possible to recover the $3 \mathrm{D}$ structure from even corrupted contact maps. In this paper we present a new hybrid technique for contact map prediction. We first predict local structural elements using an HMM. The HM$\mathrm{M}$ simultaneously represents the initiation and propagation steps of protein folding. We then apply association mining technique on top of the HMM states to predict the states that frequently co-occur with contacts. These sets are then used for predicting contacts in unseen proteins. Our model obtains $19 \%$ accuracy and coverage over the set of all proteins; the model is also 5.2 times better than a random predictor. We can significantly enhance coverage to over $40 \%$ if we sacrifice accuracy $(13 \%)$. For short proteins $($ length $<100)$ we get $30 \%$ accuracy and coverage (4.5 times better than random); if we lower accuracy to $26 \%$ we can get coverage upto $63 \%$. We believe that these results are better than (or equal to) those reported previously.

\section{Hybrid Mining Approach}

We first use an HMM to predict local substructures within the protein. We then use meta-level mining on the output of the HMM using association rule mining.

Hidden Markov Models: The description of HMM below is based on the excellent tutorial by Rabiner [7]. An HMM is a doubly stochastic process with an underlying stochastic process that is not observable (it is hidden), but can only be observed through another set of observed symbols.

An HMM is made up of a finite number $N$ of states. At each time step $t$ a new state is entered based on a transition probability distribution which depends on the previous state (the Markovian property). After each transition is made, an observation output is produced according to a fixed probability distribution which depends on the the current state. Thus there a $N$ such observation probability distributions.

An HMM is made up of the following components: $T$ is the length of the observation sequence; $N$ the number of states in the model; $M$ the number of observation symbols (for simplicity we assume here that the output is a discrete symbol, e.g. an amino acid. However we actually use a continuous vector output as we shall see later); $Q=\left\{q_{1}, q_{2}, \cdots q_{N}\right\}$ is set of HMM states; $V=\left\{v_{1}, v_{2}, \cdots, v_{M}\right\}$ is the set of output symbol$\mathrm{s} ; A=\left\{a_{i j}\right\}$ gives the set of state transition probabilities, i.e., $a_{i j}=P\left(q_{j}\right.$ at $t+1 \mid q_{i}$ at $\left.t\right) ; B=\left\{b_{j}(k)\right\}$ is the output symbol probability distribution in state $q_{j}$, i.e., $b_{j}(k)=P\left(v_{k}\right.$ at $t \mid q_{j}$ at $\left.t\right)$; and finally $\pi=\left\{\pi_{j}\right\}$ gives the initial state distribution, i.e., $\pi_{j}=P\left(q_{j}\right.$ at $\left.t=1\right)$.

Using the model, an observation sequence $O=$ $O_{1} O_{2} \cdots O_{T}$ is generated as follows: 1) choose an initial state $i_{1}$ based on $\pi, 2$ ) set position $t=1,3$ ) choose $O_{t}$ according to $\left.b_{i_{t}}(k), 4\right)$ choose $i_{t+1}$ according to $\left\{a_{i_{t} i_{t+1}}\right\}$, $i_{t+1}=1,2, \cdots N$, and 5) set $t=t+1$; return to step 3 if $t<T$; otherwise terminate the procedure.

Association Rules: The association mining [11] task can be 
stated as follows: Let $\mathcal{I}$ be a set of items, and $\mathcal{D}$ a database of examples composed of items. A subset of items is also called an itemset. The support of an itemset is the number of examples in $\mathcal{D}$ where it occurs as a subset. An itemset is frequent if its support is more than a user-specified minimum support (min_sup) value. An association rule is an expression $A \Rightarrow B$, where $A$ and $B$ are itemsets. The support of the rule is the joint probability of a example containing both $A$ and $B$. The confidence of the rule is the conditional probability that an example contains $B$, given that it contains $A$. A rule is strong if its confidence is more than a user-specified minimum confidence (min_conf). In this paper we are interested in rules with a specific item, called the class, as a consequent, i.e., we mine rules of the form $A \Rightarrow \mathbf{c}_{\mathbf{i}}$ where $\mathbf{c}_{\mathbf{i}}$ is a class attribute $(1 \leq i \leq k)$.

We mine the frequent sets based on the Formal Concept Analysis approach, which is a very elegant mathematical framework for extracting "concepts" from databases.

Consider an itemset $X$. Let $Y=\{E \in \mathcal{D} \mid X \subseteq E\}$ be the set of all examples $E$ in the database $\mathcal{D}$ where $X$ occurs. Further let $X^{\prime}=\left\{i \in \mathcal{I} \mid i \in \cap_{E \in Y} E\right\}$ be the set of all items that are common to all examples in the set $Y$. Then we say that $X$ is closed if $X=X^{\prime}$. In other words $X$ is the maximal set of items that is common to all examples in $Y$. A closed itemset is also called a concept.

The set of all closed frequent itemsets can be orders of magnitude smaller than the set of all frequent itemsets, especially for real (dense) datasets. At the same time, we don't loose any information; the closed itemsets uniquely determine the set of all frequent itemsets and their exact frequency. Thus instead of mining all the frequent itemsets we only mine the frequent closed itemsets using the CHAR$\mathrm{M}$ algorithm [12] we recently developed. A detailed description of the algorithm is beyond the scope of this paper. Suffice it to say that CHARM can handle very large diskresident or external memory databases; it has been tested on databases with millions of examples, and it scales linearly in the database size. We refer the reader to [12] for the algorithm description and its efficiency.

\section{HMMSTR: An HMM for Local Structure}

We describe here the hidden Markov model, HMMSTR [2], for general protein sequences based on the I-sites library of sequence-structure motifs [1]. In the next section we will show how we apply association mining on the output of HMMSTR to predict residue contacts.

The I-sites (Invariant or Initiation sites) library consists of an extensive set of short sequence motifs, length 3 to 19 , obtained by exhaustive clustering of sequence segments from a non-redundant database of known structures [1]. Each sequence pattern correlates strongly with a recurrent local structural motif in proteins. Approximately one third of all residues in the database are found in an I-sites motif that can be predicted with a high degree of confidence $(>70 \%)$. The library is non-redundant in that no motif is completely contained within another, longer motif. However, many of the motifs overlap. Furthermore, the isolated motif model does not capture higher order relationship$\mathrm{s}$ such as the distinctly non-random transition frequencies between the different motifs. The redundancy inherent in the I-sites model suggests a better representation that would model the diversity of the motifs and their higher order relationships while condensing features they have in common. A hidden Markov model is well suited to this purpose.

Description of HMMSTR: Each of the 262 I-sites motif was represented as a chain of Markov states, each of which contains information about the sequence and structure attributes of a single position in the motif. Adjacent positions were represented by transitions from one state to the next. Hierarchical merging of these linear chains of states, based on sequence and structure similarity, resulted in a graph containing almost all the motifs. The merged I-sites motifs comprise a network of states connected by probabilistic transitions, i.e. an HMM as shown in Figure 2.

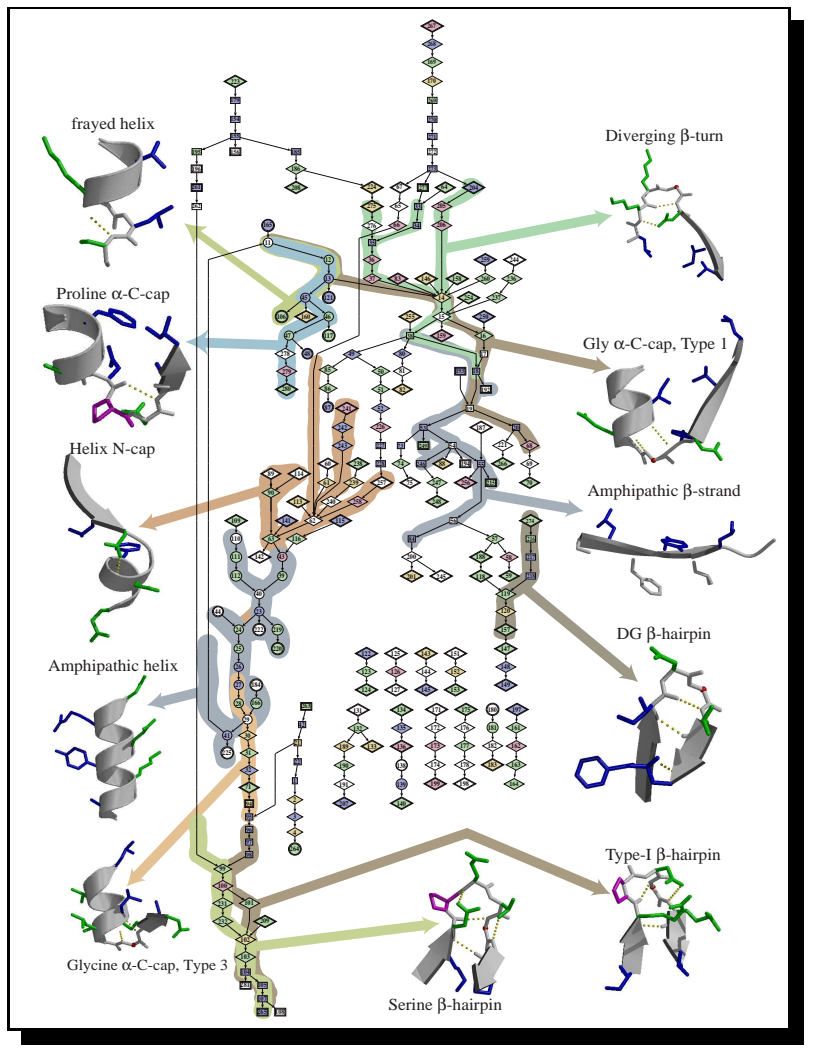

Figure 2. HMMSTR model [2]

Each HMMSTR state can produce, or "emit", amino acids and structure symbols according to a probability distribution specific to that state. There are four probability distributions defined for the states in HMMSTR, $b, d, r$, and $c$, which describe the probability of observing a particular amino acid, secondary structure, backbone angle region, or structural context descriptor, respectively. A context descriptor represents the classification of a secondary structure type according to its context. For example, a hairpin turn is distinguished from a diverging turn, and a beta-strand in the middle of a sheet is distinguished from one at the end of a sheet. More formally, for a given state $q_{i}$, there are a set of emission probabilities, collectively called $B_{i}$. The values $b_{i}(m)(m=1 \cdots 20)$ are associated with probabilities for the emission of amino acids; $d_{i}(m)(m=1 \cdots 3)$ are 
the probabilities of emitting helix $(\mathrm{H}), \operatorname{strand}(\mathrm{S})$ or loop $(\mathrm{T})$; $r_{i}(m)(m=1 \cdots 11)$ are the probabilities of emitting dihedral angle symbols, and $c_{i}(m)(m=1 \cdots 10)$ are probabilities of emitting structural context symbols.

The database is encoded as a linear sequence of amino acids and structural observables. The amino acid sequence data consists of a "parent" amino acid sequence of known three-dimensional structure, and an amino acid profile obtained by alignments to the parent sequence [1]. The amino acid of the parent sequence is denoted by $O_{t}$, and the profile by $\left\{O_{t}^{m}\right\}(1 \leq m \leq 20)$. For the structural identifiers at each position $t$, the following nomenclature is used: 3-state secondary structure $D_{t}$, discrete backbone angle region $R_{t}$, and the context symbol $C_{t}$. A sequence $s$ of length $T$ is given by the values of the attributes at all positions $s_{t}=\left\{O_{t},\left\{O_{t}^{m}\right\}, D_{t}, R_{t}, C_{t}\right\}(1 \leq t \leq T)$. A path is a sequence of states through the HMM, denoted $Q=q_{1} q_{2} \cdots q_{T}$. Thus, the probability of a sequence $s$ given the model $\lambda, P(s \mid \lambda)$, is obtained by summing the relevant contributions from all possible paths $Q: P(s \mid \lambda)=$ $\sum_{\text {all I }} \pi_{i_{1}} B_{i_{1}}\left(s_{1}\right) a_{i_{1} i_{2}} b_{i_{2}}\left(s_{2}\right) \cdots a_{i_{T-1} i_{T}} b_{i_{T}}\left(s_{T}\right)$, where $I=i_{1} i_{2} \cdots i_{T}$ is a fixed sequence of states and $B_{i}\left(s_{t}\right)$ is the probability of observing $s_{t}$ at state $q_{i}$. HMMSTR showed significant improvements in performance when we used amino acid profiles instead of single amino acids, thus $B$ is given as ( $N$ count is a global parameter): $B_{i}\left(s_{t}\right)=\left(\begin{array}{c}d_{i}\left(D_{t}\right) \\ r_{i}\left(R_{t}\right) \\ c_{i}\left(C_{t}\right)\end{array}\right) \sum_{m=1}^{20} b_{i}(m)^{N \text { count } \times O_{t}^{m}}$

Training HMMSTR: For training and testing of the $\mathrm{H}-$ MMSTR we used a non-redundant database of proteins of known structure, PDBselect:December 1998 [4] containing 691 proteins and their sequence families. The proteins in the set have $<25 \%$ sequence similarity. Entries in the database were selectively removed if the structure was solved by $\mathrm{N}$ MR, had a large number of disulfide bridges or cis-peptide bonds, or if it was a membrane-associated protein according to the header records. Disordered or missing coordinates in the middle of a sequence were addressed by dividing the sequence at that point. Contiguous segments of length less than 20 were ignored. Multiple sequence alignments were generated from each sequence using PSI-BLAST after filtering the query sequences for low-complexity regions. Data for training the HMM included the sequence profile, computed from the multiple sequence alignment as described before [1], the DSSP secondary structure assignments [5], the backbone angles, and a structural "context" symbol.

Backbone angles were measured from the coordinates and assigned, using a Voronoi method, to 11 regions of phi/psi space. The centroids of 10 regions were chosen by $\mathrm{K}$-means clustering of a large subset of trans phi/psi pairs from the database. The 11 th region is all cis peptides.

A randomly selected set of 73 of the 691 proteins $(19,000$ positions) was then set aside and not used for training, but only for the final cross-validation. Before cross-validation, a test for true independence was applied to each member of the test set, and 12 members were removed. The final test set thus contained 61 proteins and 16,000 positions.

The remaining set of 618 parent sequences $(145,000$ po- sitions) was used for training, and divided into a large set of 564 sequences (133,000 positions), used for optimization via the Expectation-Maximization algorithm, and a small set of 54 sequences (12,000 positions) used to evaluate the predictive ability of the model during training. Note that the small set of 54 sequences is used only for evaluation of the performance of a model and may thus appear to be a test set. However, decisions regarding the modification of the model are based on results of those evaluations. The set of 54 sequences is therefore not a test set, but a training set. For the final round of training we re-combined the large and small training sets, to a total of 618 sequence families. After the final round of training, the models were frozen.

\section{Data Format and Preparation}

After HMMSTR is built we again took the 691 proteins from PDBSelect and computed for each protein the optimal HMMSTR states that agree with the observed amino acids in the protein. In other words for each protein sequencestructure we solve the estimation problem, i.e., given the observation sequence $O=O_{1} O_{2} \cdots O_{T}$, how to choose a state sequence $I=i_{1} i_{2} \cdots i_{T}$, which is optimal. The output probability distributions of all the states thus chosen for a protein sequence is used as input for the association mining algorithm. In fact, rather than a single state associated with a given residue, we have available the probability that the residue at the given position is associated with all the states of HMMSTR, i.e., we have available $P\left(q_{i} \mid a_{j}\right)$ for all the 282 HMMSTR states $(1 \leq i \leq 282)$ for all the residues in a given protein $(1 \leq j \leq n$, where $n$ is the length of the protein). For each residue we also know the amino acid at that position; the $b, d, r$, and $c$ outputs, which describe the probability of observing a particular amino acid, secondary structure, backbone angle region, or structural context descriptor, respectively; the spatial coordinates of the $\alpha$-Carbon atom $\langle x, y, z\rangle$; a distance vector of length $n$ giving the distance of this residue from all other residues in the protein; and the 20 amino acid profiles for that position. A protein data file may look like this:

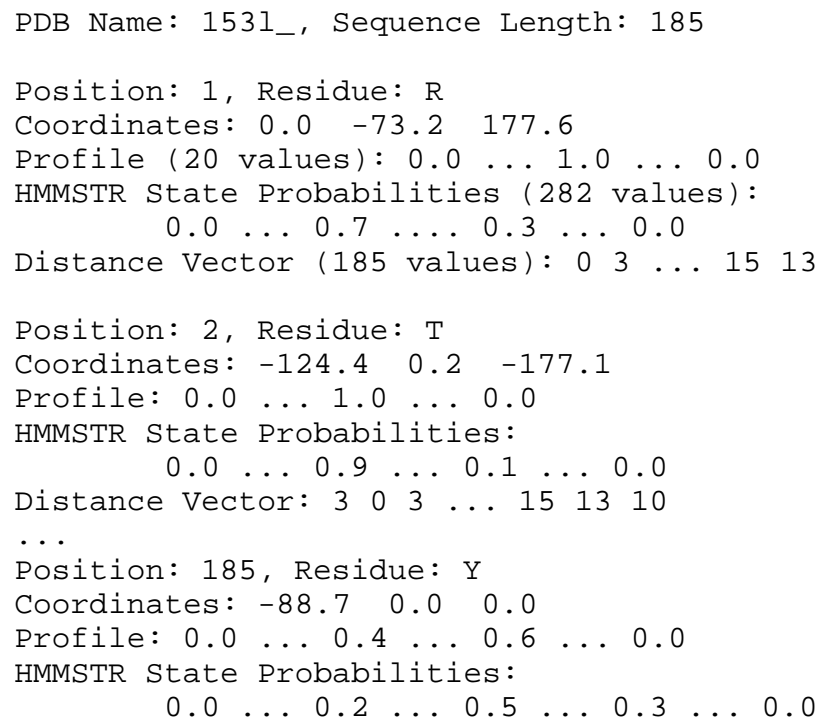


Distance Vector: $1513 \quad 10 \ldots 5$ 5 30

We have one file for each of the 691 proteins from PDBSelect. Disordered or missing coordinates in the middle of a protein sequence were addressed by dividing the sequence at that point. This produces a set of 794 files, most of them containing an entire protein sequence, but some of these correspond to proteins that were split.

Given a protein file, we now have to transform the data into a format that can be easily mined for frequent closed itemsets, i.e., we need to prepare the data in the relational or tabular format where we have multiple attributes (columns) for each example (rows) or record. Since we are interested in predicting the contact between a pair of amino acids, we use each pair as an example in the training set, associated with a special class attribute indicating whether it is a contact $(C)$ or non-contact $(N C)$; amino acids $a_{i}$ and $a_{j}$ are in contact if $\delta\left(a_{i}, a_{j}\right)<7 \AA$, i.e., the distance between $\alpha$-carbons of amino acids $a_{i}$ and $a_{j}$ is less then $7 \AA$. Our new database has an entry showing the two amino acids and their class for each pair of amino acids for each protein. In order to avoid predicting purely local contacts we ignore all pairs whose sequence separation $|i-j|<4$. Note also that the number of contacts $N_{C}$ is a lot smaller than the number of non-contacts $N_{N C}$ for any protein.

We found that the percentage of contacts (or number of database entries with class 1) over all pairs is less than $1.7 \%$. Across the 794 files, the longest sequence had length 907, while the smallest had length 35 . There were 17,618,115 pairs over all proteins, while only 292,126 pairs were in contact. This database thus corresponds to a highly biased binary classification problem. That is, we have to build a mining model that can discriminate between contacts and non-contacts between amino acids pairs, where the examples are overwhelmingly biased towards the non-contacts.

Our database so far doesn't have enough information for good discrimination. All we have is the amino acids making up the pair and whether they are in contact or not. We need to add more "context" information to facilitate the classification. It is easy to incorporate, for each amino acid in the pair, the 3 secondary structure symbols $\left(d_{i}, d_{j}\right)$, the 11 backbone angle regions $\left(r_{i}, r_{j}\right)$, and the 10 structural context descriptors $\left(c_{i}, c_{j}\right)$. For each pair we would also like to add the HMMSTR state probabilities. Since association rules only work for categorical attributes, we need to convert the continuous state probabilities into discrete values. To do this we take the ratio of each of the 282 HMMSTR state probabilities for $a_{i}$ against the background or prior probability of an amino acid being in that state; if the ratio is more than some threshold we include the state in the context of $a_{i}$, else we ignore it. We repeat the same process for $a_{j}$. Using a similar thresholding method one can incorporate the amino acid profiles for positions $i$ and $j$. With all this context information for both $a_{i}$ and $a_{j}$ we obtain a new database to be used to find the frequent itemsets characterizing the contacts and non-contacts. This has the following columns for pairs of amino acids over all proteins:

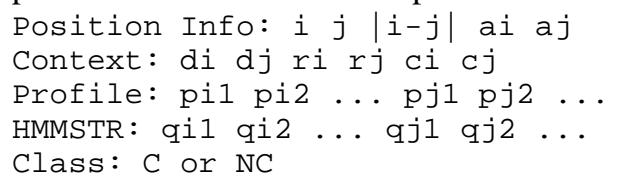

Note that the number of columns can be variable for different pairs depending on the profile and HMMSTR state probabilities. $p_{i_{1}}, p_{i_{2}}$, etc. show the other amino acids that can appear in position $i$ (provided the probability is more than some threshold), and finally $q_{i_{1}}, q_{i_{2}}$, etc. show HMMSTR states with probabilities more than some factor of the prior probability of those states.

\section{Association Mining on the Pairs Database}

We are now in a position to cast the above database in the association framework. Each attribute-value pair is an item, and is represented with a fixed, unique integer. For example $a_{i}=G$ is one item and $a_{i}=L$ is another item. By the same token each value of $c_{i}, d_{i}, r_{i}, L_{i}$, and $R_{i}$ is a different item. Each of the HMMSTR states becomes a distinct item, as do the profile values. The items for the context attributes of $a_{i}$ and $a_{j}$ are also kept distinct. Finally we separate the examples that are contacts from those that are non-contacts to get two databases, denoted as $\mathcal{D}_{C}$ and $\mathcal{D}_{N C}$, respectively.

Our goal is to find high support and high confidence rules of the form $A \Rightarrow C$ and $A \Rightarrow N C$, that discriminate between the contact pairs and the non-contact pairs, respectively. Below we describe the mining/training and testing phases, where we learn from examples using the frequent closed itemsets, and then classify unseen examples as being contacts or non-contacts, respectively.

\subsection{Mining on Known Examples}

The goal of the mining phase is to learn from known contact and non-contact examples and build a model or rule set that discriminates between the two classes. We selected a random $90 \%$ of the files for training, out of a total of 794 files. The remaining $10 \%$ of the files were kept aside for testing the mined rule set. Since we are primarily interested in predicting the contacts rather than the non-contacts, we mine only on the contacts database $\mathcal{D}_{C}$. However, we do use the non-contacts database $\mathcal{D}_{N C}$ to prune out those itemsets that are frequent in both sets. Building a discriminative rule set consists of the following steps, in order:

Mining: We use CHARM [12] to mine all the frequent closed itemsets in $\mathcal{D}_{C}$ based on a suitably chosen min_sup value. Let's denote the set of frequent closed itemsets as $\mathcal{F}$. Counting: We compute the support of all itemsets in $\mathcal{F}$ in the non-contacts database $\mathcal{D}_{N C}$.

Pruning: We compute the probability of occurrence of each itemset in $\mathcal{F}$ in both the contact and non-contact databases. The probability of occurrence is simply the support of the itemset divided by the number of examples in the given dataset. For example, if itemset $X \in \mathcal{F}$, then the probability of its occurrence in $\mathcal{D}_{C}$ is given as $P\left(X, \mathcal{D}_{C}\right)=$ $\sigma\left(X, \mathcal{D}_{C}\right) /\left|\mathcal{D}_{C}\right|$. As a first step in pruning we can remove all itemsets $X \in \mathcal{F}$ which have a greater probability of occurrence in the non-contact database than in the contact database, i.e., if $P\left(X, \mathcal{D}_{N C}\right)>P\left(X, \mathcal{D}_{C}\right)$. Actually, we compute the ratio of the contact probability versus the noncontact probability for $X$, and prune it if this ratio is less than some suitably chosen threshold $\rho$, i.e., we prune $X$ if $P\left(X, \mathcal{D}_{C}\right) / P\left(X, \mathcal{D}_{N C}\right)<\rho$. In other words we want to retain only those itemset that have a much greater chance of predicting a contact rather than a non-contact. 


\subsection{Testing on Unknown Examples}

The goal of testing is to find how accurately the mined set of rules predict the contacts versus the non-contacts in new examples not used for training. We used a random $10 \%$ of the files in the database for testing. The test set had a total of 2,336,548 pairs, out of which 35,987 or $1.54 \%$ were contacts. Since we do know the true class of each example it is easy for us to find out how well our rules are for prediction. For testing we generate a combined database $\mathcal{D}_{t}$ containing all pairs of amino acids in contact or otherwise. For each example we know the true class. We assign each example a predicted class using the following steps:

Evidence Calculation: For each example $E$ in the test dataset $\mathcal{D}_{t}$, we compute which itemsets in the set of mined and pruned closed frequent itemsets $\mathcal{F}$ are subsets of $E$. Let's denote the set of these itemsets as $S$. We next calculate the cumulative contact and non-contact support for example $E$, i.e., the sum of the supports of all itemsets in $S$ in the contact and non-contact database. Finally, we compute the evidence for $E$ being a contact, i.e., the ratio of the cumulative contact support over non-contact support, denoted as $\rho_{E}$. Any $E$ with zero contact support is taken to be a non-contact and discarded, and only the examples or with positive contact support are retained for the next step.

Prediction: To make the final prediction if a test pair of residues is in contact or not, we sort all test examples $E$ (with positive cumulative contact support) in decreasing order of contact evidence $\rho_{E}$. Finally, the top $\gamma$ fraction of examples in terms of $\rho_{E}$ are predicted to be contacts and the remaining $1-\gamma$ fraction of examples as non-contacts. How $\gamma$ is chosen will be explained below.

\subsection{Model Accuracy and Coverage}

In predicting contacts versus non-contacts for the test examples, we have to evaluate the mined model based on two metrics: Accuracy and Coverage. Furthermore, we are only interested in the prediction of contacts; thus accuracy and coverage is only considered for contacts. Accuracy is the ratio of correct contacts to the predicted contacts, while coverage is the percentage of all contacts correctly predicted. Thus, accuracy tells us how good the model is, while coverage tells us the number of contacts predicted.

More formally, let $N_{t c}$ denote the number of true contacts in the test examples, $N_{p c}$ the number of predicted contacts, $N_{t p c}$ the number of true predicted contacts, and let $N_{a}$ denote the number of all possible contacts, i.e., $N_{a}=(N-3) \times(N-2) / 2$ (where $N$ is the protein length), since the contact map is symmetric and pairs with sequence separation less than 4 are ignored. The accuracy of the model is given as $A=N_{t p c} / N_{p c}$, and the coverage of the model is given as $C=N_{t p c} / N_{t c}$ We also compare our model against a random predictor. The accuracy of random prediction of contacts is defined as $A_{r}=N_{t c} / N_{a}$.

The number of contacts predicted $N_{p c}$ of course depends on how we chose $\gamma$, since the top $\gamma$ fraction of test examples based on evidence is predicted as contacts. Since a protein is characterized by $N_{t c}$ true contacts, we set $\gamma=N_{t c}^{*} / N_{a}^{*}$ and then predict the top $\gamma$ fraction of examples as contacts. Note that $N_{t c}^{*}$ and $N_{a}^{*}$ denote the actual contacts and all pairs, respectively, that have positive contact support, since we discard examples with zero contact support. By adopting the above method, the number of predicted contacts is limited to those actually present in the protein. Further, this method has been used by previous approaches to contact map prediction $[3,6]$; we use it for comparison.

\section{Experimental Results}

We mined the pairs database using various combinations of context information and then tested the model on the unseen proteins. The pairs databases for training and testing had the following approximate sizes: $\mathcal{D}_{C}=32 M B$ for the training contacts database, $\mathcal{D}_{N C}=2 G B$ for the training non-contacts database, and $\mathcal{D}_{t}=340 M B$ for the testing database (includes both contacts and non-contacts). For all experiments below, we used a minimum support of $0.5 \%$ in the contact database, and we pruned a pattern if the ratio of contact to non-contact frequency was less than 4 (except for the amino acids only case where we used a ratio of 1.5).

Amino Acids Only: Our first goal was to test how much information is contained in the amino acids only, i.e., for both training and testing, each example consisted of only the two amino acids $a_{i}$ and $a_{j}$, and nothing else. Figure 3 shows the accuracy, coverage, and improvement of the mined model over the random predictor for the test set. The accuracy and coverage is the mean value over all proteins. The figure shows that the amino acids have some information that can be used to predict contacts versus non-contacts, but this information is not too good. The figure plots the accuracy and coverage as percentages. It also plots the improvement of the model over the random predictor. The $\mathrm{x}$-axis shows the prediction factor, which is related to the $\gamma$ value (used to predict the top fraction of pairs as contacts). The prediction factor is in multiples of $N_{t c}^{*}$, the number of true contacts in the protein with positive contact evidence. For example, a value of 10 means that the top $\left(10 \times N_{t c}^{*}\right) / N_{a}^{*}$ fraction of the examples are predicted as contacts.

The left-most graph in Figure 3 shows the accuracy and coverage of the predictor over test proteins of all lengths. The other two figures on the right show how accuracy and coverage change with protein length. We have divided the test proteins into four bins: $1 \leq N<100,100 \leq N<170$, $170 \leq N<300$, and $300 \leq N$.

We find that over all proteins the amino acids by themselves can be used to give an $8.5 \%$ accuracy, $1.5 \%$ coverage, and an improvement over a random predictor by a factor of 2.4. Note also the interesting trend in the graph. As the prediction factor increases we get better and better coverage, but the accuracy trails off. This represents the classic accuracy versus coverage trade-off common to many prediction problems. Which value to choose for the prediction factor depends on what is more important. It has been reported in [9] that the 3D structure of proteins can be recovered quite robustly, even from corrupted contacts maps. This implies that coverage should have an higher weight than accuracy. In any case, if we had to choose a value representing the best trade-off, we can pick the point where the accuracy and coverage curves intersect. This happens for a prediction factor of 7 , where we have roughly $7 \%$ accuracy 

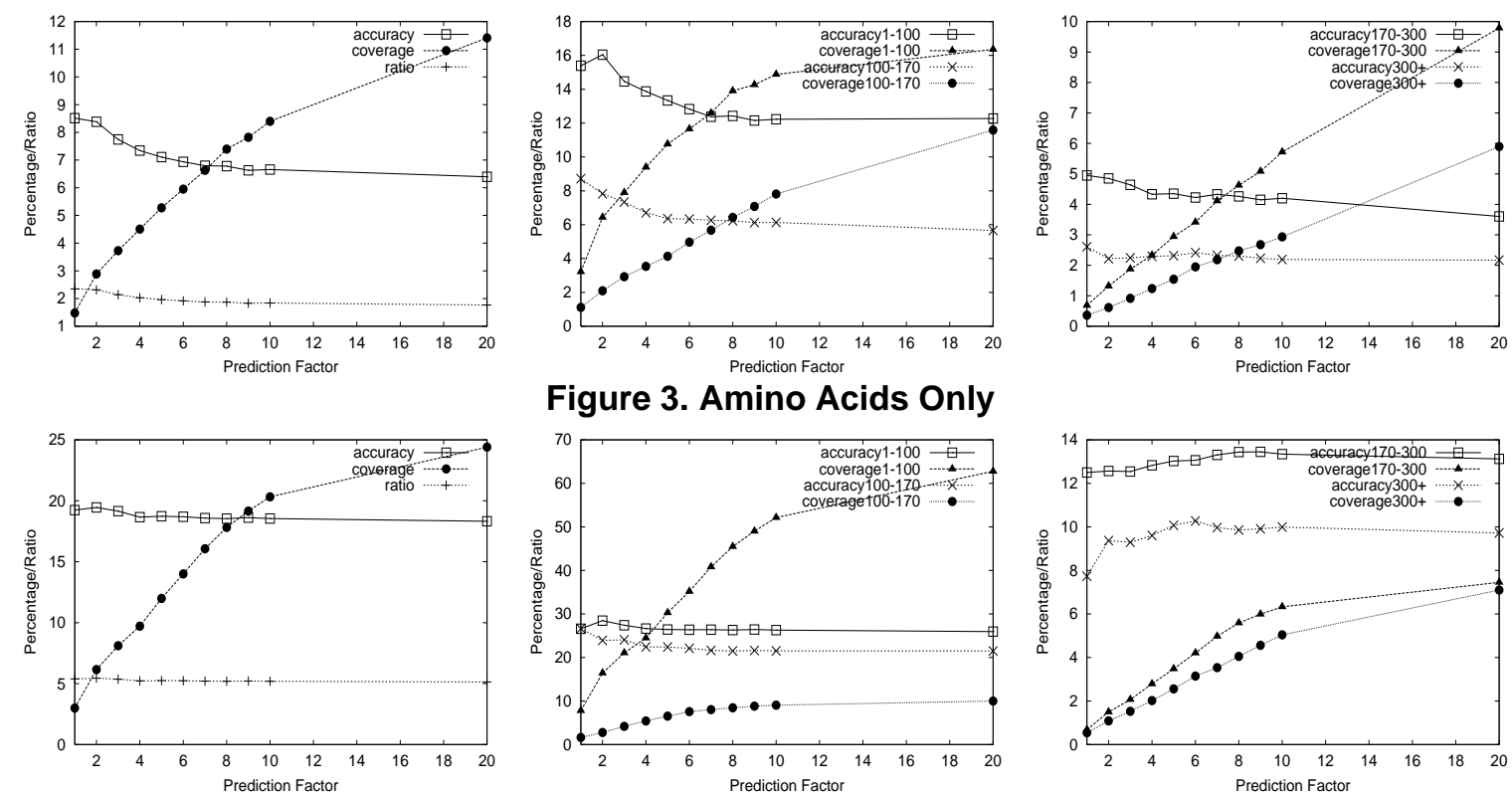

Figure 3. Amino Acids Only
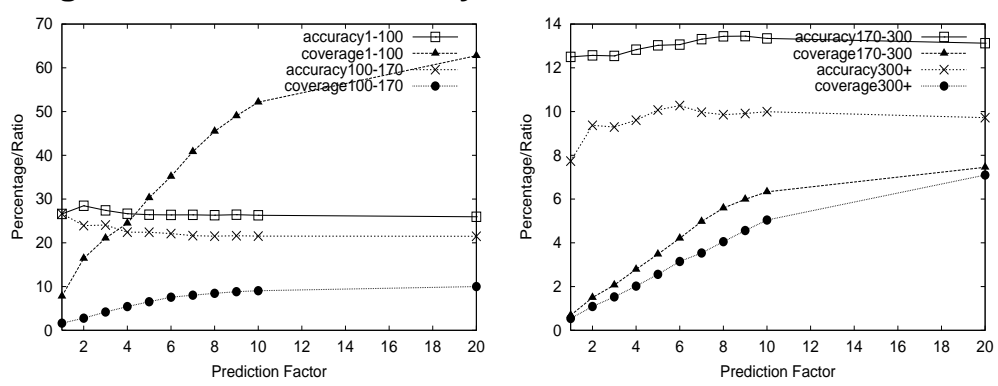

Figure 4. HMMSTR States and Amino Acids
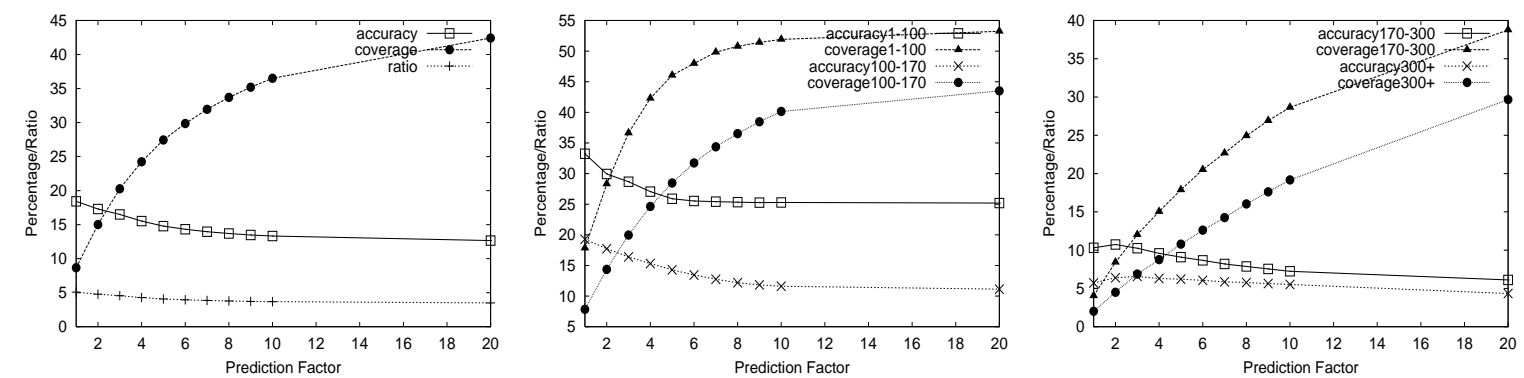

Figure 5. HMMSTR States, Amino acids, and $R_{t}, D_{t}, C_{t}$ Symbols
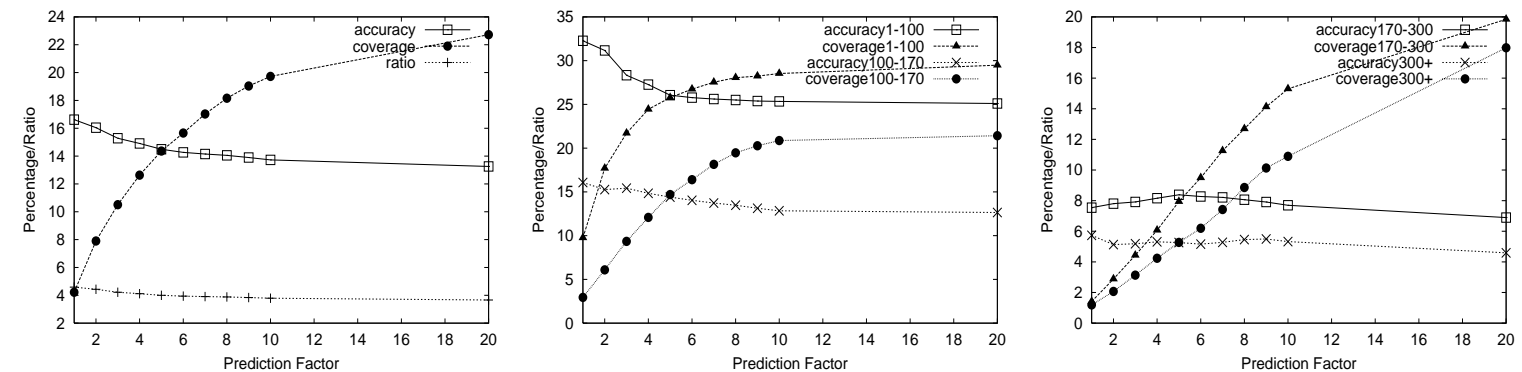

Figure 6. HMMSTR States, Amino Acids, and Amino Acid Profiles

and coverage, and which is 2 times better than random. For $6.3 \%$ accuracy we can increase coverage to $14 \%$.

When we consider the results for proteins of different lengths, we find the same trade-off between accuracy and coverage. Looking at the crossover point, we get around $13 \%$ accuracy and coverage for short proteins with $N<$ $100,6 \%$ for $100 \leq N<170,4.5 \%$ for $170 \leq N<300$, and around $2 \%$ of longer proteins.

HMMSTR States and Amino Acids: We next added the HMMSTR states corresponding to $a_{i}$ and $a_{j}$, i.e., we added the columns $q_{i_{1}}, q_{i_{2}}, \cdots$ and $q_{j_{1}}, q_{j_{2}}, \cdots$ to the training and testing sets. Figure 4 shows the results. If we look at the cross-over point we get almost $19 \%$ accuracy and coverage, while the model remains 5.2 times better than random.
For $18 \%$ accuracy we can get coverage of $25 \%$ (still 5.1 times better than random). Figure 7 shows the results in a slightly different format. It plots the improvement in coverage/accuracy over a random prediction. These results are comparable to or better than the results recently reported in [13], where they examined pairwise amino acid interaction$\mathrm{s}$ in the context of secondary structural environment (helix, strand, and coil), and used the environment dependent contact energies for contact prediction experiments. For about $25 \%$ coverage our model does more than 5 times the random predictor, as compared to the 4 times improvement reported in [13]. Figure 8 shows the predicted contact map for the protein $2 i g d$ that we used in the introduction. We got $35 \%$ accuracy and $37 \%$ coverage for this protein. The fig- 


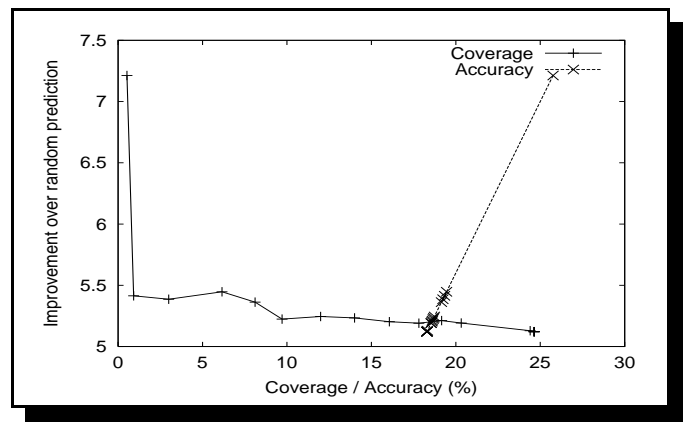

Figure 7. Improvement over Random

ure shows the true contacts, the contacts correctly predicted, and all the contacts predicted.

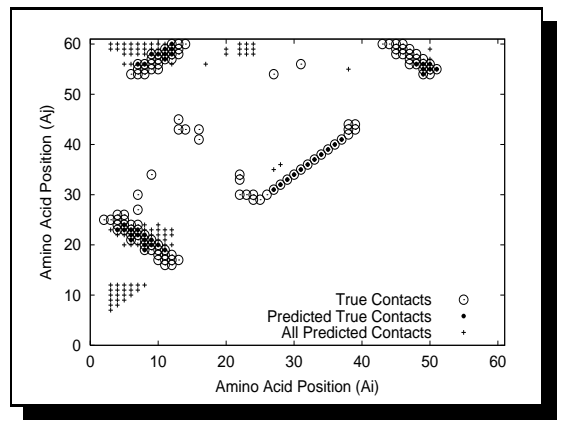

Figure 8. Predicted Contact Map (2igd)

If we look at proteins of various lengths in Figure 4, we find that for $N<100$, we get $26 \%$ accuracy and $63 \%$ coverage at the extreme point ( 4 times over random). For $100 \leq N<170$ we get $21.5 \%$ accuracy and $10 \%$ coverage towards the end (6 times over random), for $170 \leq N<300$ we get $13 \%$ accuracy and around $7.5 \%$ coverage $(6.5$ times over random), and for longer proteins we get $9.7 \%$ accuracy and $7.5 \%$ coverage ( 7.8 times over random). We believe these results are the best, or at least comparable to those reported so far in the literature on contact map prediction [3, 6]. For example, Fariselli and Casadio [3], used a Neural Network based approach over pairs database, with other contextual information like sequence context windows, amino acid profiles, and hydrophobicity values. They reported an $14.4 \%$ accuracy over all proteins, with an 5.4 times improvement over random. They also got $18 \%$ accuracy for short proteins with an 3.1 times improvement over random. Olmea and Valencia [6] on the other hand used correlated mutations in multiple sequence alignments for contact map prediction. They added other information like sequence conservation, alignment stability, contact occupancy, etc. to improve the accuracy. They reported $26 \%$ accuracy for short proteins, but did not report results for all proteins. While we believe that our hybrid approach does better, we should say that direct comparison is not possible, since previous works used a different (and smaller) PDB_select database for training and testing. One draw back of these previous approaches is that they do not report any coverage values, so it is not clear what percentage of contacts are correctly predicted. Another approach to contact map prediction was presented in [8], which was based on correlated mutations. They obtained an accuracy of $13 \%$ or 5 times better than random.

Adding Additional Information: We next tried to add more columns to the training database. For example we separately added the amino acid profiles, and the structural context symbols for the 3 -state secondary structure $D_{t}$, discrete backbone angle region $R_{t}$, and the context symbol $C_{t}$. The results for these cases are shown in Figure 5 and Figure 6. As we can see adding the profiles did not add any additional prediction power to our model, while adding the structural symbols had a positive (somewhat mixed) effect on accuracy and coverage. It appears that while the accuracy of the prediction drops a little there is tremendous boost in the coverage of the model. For example at around $18 \%$ accuracy we get about 25\% coverage using the HMM states and amino acids (see Figure 4), but when we add the structural symbols, we get about $44 \%$ coverage for an accuracy of $12.5 \%$. This tells us that the structural symbols can be helpful in providing the right context and thus help in identifying a larger portion of the contacts.

In conclusion we have presented a new hybrid HMM and association rule mining method for contact predictions. Our results are the best or comparable to those previously reported. We are currently working to further improve both accuracy and coverage by carefully selecting many of the threshold parameters used in the experiments, as well as by adding additional attributes that might help prediction.

\section{References}

[1] C. Bystroff and D. Baker. Prediction of local structure in proteins using a library of sequence- structure motifs. Journal of Molecular Biology, 281(3), 565-77, 1998.

[2] C. Bystroff, V. Thorsson, and D. Baker. HMMSTR: A hidden markov model for local sequence-structure correlations in proteins. Journal of Molecular Biology, (to appear), 2000.

[3] P. Fariselli and R. Casadio. A neural network predictor of residue contacts in proteins. Protein Engg, 12(1):15-21, '99.

[4] U. Hobohm and C. Sander. Enlarged representative set of protein structures. Protein Science, 3(3), 522-524, 1994.

[5] W. Kabsch and C. Sander. Dictionary of protein secondary structure: pattern recognition of hydrogen-bonded and geometrical features. Biopolymers, 22, 2577-2637, 1983.

[6] O. Olmea and A. Valencia. Improving contact predictions by the combination of correlated mutations and sources of sequence info. Folding \& Design, 2, S25-S32, June' 97.

[7] L. Rabiner. A tutorial on HMMs and selected applications in speech recognition. Proc. of IEEE, 77(2):257-86, 1989.

[8] D. Thomas, G. Casari, and C. Sander. The prediction of protein contacts from multiple sequence aligments. Protein Engineering, 9(11):941-48, 1996.

[9] M. Vendruscolo, E. Kussell, and E. Domany. Recovery of protein structure from contact maps. Folding \& Design, 2(5), 295-306, Sept. 1997.

[10] Q. Yi et al. Prediction and structural characterization of an independently folding substructure in the src sh3 domain. Journal of Molecular Biology, 283(1), 293-300, 1998.

[11] M. J. Zaki. Scalable algorithms for association mining. IEEE Trans Knowledge \& Data Engg, 12(3):372-390, 2000.

[12] M. J. Zaki and C.-J. Hsiao. CHARM: An efficient algo. for closed association mining. CS TR-99-10, RPI, Oct 1999.

[13] C. Zhao and S.-H. Kim. Environment-dependent residue contact energies for proteins. Proc. Natl. Acad. Sci. USA, 97(6), 2550-5, 2000. 